\title{
The Relationship Between University Students' Nutritional Status, Cardio-Metabolic Biomarkers and Physical Activity Levels
}

\author{
Hayrunisa Icen ${ }^{1}$, Fatma Esra Gunes ${ }^{2}[$ \\ ${ }^{1}$ Marmara University, Institue of Health Sciences, Istanbul, Turkey \\ ${ }^{2}$ Marmara University, Faculty of Health Sciences, Istanbul, Turkey \\ Correspondence Author: Hayrunisa Icen \\ E-mail: hayrunnisaicen@hotmail.com
}

Received: 03.11.2020 Accepted: 09.03.2021

\begin{abstract}
Objective: This study aimed to evaluate the relationship between nutritional status, cardiometabolic biomarkers and physical activity levels in university students.

Methods: Firstly, fasting plasma total cholesterol, triglyceride, high-density lipoprotein cholesterol (HDL-C) levels were analyzed from participants' blood samples, and the homeostatic model assessment of insulin resistance (HOMA-IR) values were calculated after measuring fasting plasma glucose and insulin. Participants' weight, height, percentage of body fat, waist and hip circumference were measured and body mass index (BMI) was calculated. The International Physical Activity Questionnaire (IPAQ)and a questionnaire on socio-demographic characteristics were applied to participants. Energy and nutrient intakes were calculated from participants'24-hour dietary recall records. The statistical analysis of data was performed with SPSS version 21 software. For statistical significance, the total type-1 error level was determined as $5 \%$.

Results: Most of the participants' energy, dietary fibre, vitamins D and B1, folate, calcium and iron intakes were below recommended levels. HDL-C level was shown to be negatively correlated with total fat and saturated fatty acid intake $(p<0.05)$. Omega- 3 intakes of participants were negatively correlated with fasting plasma insulin and HOMA-IR ( $p<0.05)$. HDL-C was negatively correlated with BMI and waist-to-hip ratio, while fasting plasma insulin, triglyceride levels and HOMA-IR values were positively correlated with them $(p<0.05)$. According to the IPAQ, it was found that $15.9 \%$ of participants had a low level of physical activity and $66.1 \%$ of them had a moderate level of physical activity.

Conclusion: It is concluded that providing nutrition education and counselling services to students, improving campus facilities for physical activity are necessary for cardiometabolic health.
\end{abstract}

Keywords: University students, nutritional status, biomarkers, exercise, health

\section{INTRODUCTION}

Nowadays, health-threatening and changeable behaviours such as inadequate and imbalanced nutrition, sedentary lifestyle, tobacco/smoking and excessive alcohol consumption, which are frequently observed among young people, are among the risk factors of various chronic diseases (1). It is thought that the university period is a critical period in which lifelong habits are formed and may have a permanent effect on the development of chronic diseases (2).

It is known that biomarkers including weight, body mass index (BMI), waist circumference (WC), blood pressure, lipid profile and glycemic status are used to determine cardiometabolic risk $(3,4)$. The homeostatic model assessment (HOMA) used in determining insulin resistance (IR) has been proven to be a safe tool (5). Assessment of body composition by various anthropometric measurements is generally used to determine the health status of the population. The data of body fat percentage (body fat\%), WC, waist-to-hip ratio (WHR) are reported more sensitive than $\mathrm{BMI}$, which is frequently used to determine the risks of metabolic and cardiovascular diseases (6).

Adequate and balanced nutrition can be defined as providing the right nutritional habits, the number of daily meals and menu diversity, and thus meeting the requirements for energy and nutrients for healthy development (7). Behaviours that negatively affect health, such as insufficient food intake and physical inactivity, are frequently observed in university students $(8,9)$. They are considered to be in the risk group of nutritional deficiency due to these unhealthy eating behaviours.It is known that a dietary model characterized by high-calorie intake due to high consumption of fat and saturated fat, sugar, alcohol and fast food and insufficient consumption of vegetables and fruits, monounsaturated fatty acids (MUFA), polyunsaturated fatty acids (PUFA) and fish is widely observed in this population. Besides, inadequate intake of dietary fibre, folate, calcium, iron and vitamin A has been reported in this population (10). 
It is predicted that unhealthy eating habits will increase the risk of obesity, metabolic and cardiovascular diseases (10, 11).It is known that inadequate and unbalanced nutrition pattern, which is common in university students, causes atherogenic dyslipidemia, endothelial dysfunction and insulin resistance, and increases the risk of coronary heart disease, inflammation and hepatic fat synthesis in the body (12). Therefore, before cardiovascular and metabolic diseases appear clinically, it is important to evaluate cardiometabolic health with biomarkers such as fasting plasma glucose, insulin, triglyceride, HDL-C and various anthropometric measurements that are affected by dietary habits (13).

Physical activity, which is a globally accepted health promotion means, constitutes an essential factor of public health policies in both developed and developing countries (14). A meta-analysis reported that a rapid decrease in the level of physical activity is observed between the university students with ages of 18-24 (8). An increased sedentary lifestyle is a risk factor for early death and various chronic diseases such as type 2 diabetes, metabolic syndrome and cardiovascular diseases, and this increase is at a higher level in university students compared to the general young population (15).

In this study, it was aimed to evaluate the relationship between university students' nutritional statuses, cardiometabolic biomarkers and physical activity levels.

\section{METHODS}

This cross-sectionalstudy was carried out with 422 volunteer students studying at Marmara University between January 2018 and March 2020. Approval from Marmara University Faculty of Medicine Ethics Committee and necessary permissions from faculties were obtained for the study (Protocol Number-Date: 09.2017.044 - 06.01.2017).

Participants who are well-communicated and volunteer and do not have any chronic disease, pregnancy, continuous medication, medical nutritional therapy were informed about the study and signed a voluntary consent form. In addition to a questionnaire on socio-demographic characteristics, the short form of the IPAQ was applied to the participants by face-to-face interview technique. MET (metabolic equivalent task) obtained by evaluating the IPAQ, is a physiological measure expressing the energy cost of physical activity and is defined as the ratio of metabolic rate during a specific physical activity to a reference metabolic rate(16).The total physical activity score (MET - min/week) was calculated by multiplying the intensive, moderate activity and walking times with the specified coefficients and converting to MET (metabolic equivalent). Accordingly, the physical activity level of the participants was determined (17).

The 24-hour dietary recall (24HDR) was taken from the participants by the researcher. Energy and nutrient intakes were calculated using the Nutrition Information System (BeBiS 8) program from 24HDR records. According to the recommends of the individuals with the ages of 19-30 stated in the Turkey Specific Food and Nutrition Guide (TSFNG) (18), the status of meeting the energy and nutrient requirements of the participants were determined.

In the morning, after at least 12 hours of fasting the blood samples were taken from the participants. Yellow capped plastic $5 \mathrm{~mL}$ BD Vacutainer ${ }^{\circ} \mathrm{SST}^{\mathrm{TM}}$ gel tubes were used for blood samples to be serum separated. After the blood samplestaken into biochemistry tubes were centrifuged at $4000 \mathrm{rpm}$ for 10 minutes, their serums were separated and stored in a deep freezer at $-20^{\circ} \mathrm{C}$ until analysis. And thenthey were taken to the clinical laboratory to be analyzed for fastingplasma total cholesterol, triglyceride, HDL cholesterol, insulin and glucose levels without breaking the cold chain.

After blood samples were taken, the body weight and body fat percentage ofthe participants were measured with the Inbody 120 device, which performs bioelectrical impedance analysis (BIA). The height measurement was with a $0.1 \mathrm{~cm}$ sensitive stadiometer. WC and hip circumference were measured by an experienced researcher with a non-flexible tape measure.Also, WHR (WC divided by hip), BMI (weight in kilograms divided by the square of height in metres) and HOMA-IR [(fasting plasma glucose level $(\mathrm{mg} / \mathrm{dL}) \times$ fasting plasma insulin level $(\mu \mathrm{lU} / \mathrm{mL})) / 405$ ] were calculated.The cutoff point of 2.5 was used to separate participants into normal or having IR (5).According to the World Health Organization (WHO), a healthy WHR should be 0.9 or less in men and 0.85 or less for women.

Statistical analysis of the datawas carried out using SPSS version 21 software. The normality of variables was analyzedusing the Kolmogorov-Smirnov test and the histogramgraph. Discrete data are stated as number (n) and percentage (\%) distributions, while continuous data are expressed as median and interquartile range (IQR) values. Correlations were determined by using the Spearman test. The Pearson Chi-Square $\left(X^{2}\right)$ test was used to compare qualitative variables. For statistical significance, the total type-1 error level was determined as $5 \%$.

\section{RESULTS}

The general characteristics of the participants are provided in Table 1. It was shown that $86 \%$ of the participants were female, the averageage and the median BMI values were $20.6 \pm 1.78$ years and $21.3 \mathrm{~kg} / \mathrm{m}^{2}$, respectively. According to the $\mathrm{BMI}$ classification of the WHO, $70.1 \%$ of the participants were determinednormal and $14.1 \%$ pre-obese and obese. Students studying in the Department of Nutrition and Dietetics (ND) constitute $61.8 \%(n=261)$ of the participants and $38.2 \%(n=161)$ of those studying in other departments. First-year students constitute $41.2 \%(n=174)$ and the last year (4th, 5th and 6th grade) students constitute $13.5 \%$ $(n=57)$ of the participants. It was observed that $15.9 \%$ of the participants had a low, $66.1 \%$ moderate, and $18 \%$ high physical activity level. The median MET-score of the participants was found 1512.0. Participants, had a family history of heart disease, made up $22.3 \%$ of the group. And, participants using 
omega-3 supplements made up only $6.2 \%$ of the group. It was observed that tobacco smoking and alcohol consumption frequency was $14.7 \%, 13.7 \%$, respectively. Also, according to the HOMA-IR, participants who have IR were found $18 \%$ of all of them.

Table 1. General characteristics of the participants

\begin{tabular}{|c|c|c|c|c|c|c|}
\hline \multirow[b]{2}{*}{ General characteristics } & \multicolumn{2}{|c|}{ Female $(n=363)$} & \multicolumn{2}{|c|}{ Male $(n=59)$} & \multicolumn{2}{|c|}{ Total $(n=422)$} \\
\hline & $\mathrm{N}$ & $\%$ & $\mathbf{N}$ & $\%$ & $\mathrm{~N}$ & $\%$ \\
\hline \multicolumn{7}{|l|}{ Department } \\
\hline Nutrition and Dietetics & 244 & 67.2 & 17 & 28.8 & 261 & 61.8 \\
\hline Other departments & 119 & 32.8 & 42 & 71.2 & 161 & 38.2 \\
\hline \multicolumn{7}{|l|}{ Physical activity level } \\
\hline Low & 59 & 16.3 & 8 & 13.6 & 67 & 15.9 \\
\hline Moderate & 248 & 68.3 & 31 & 52.5 & 279 & 66.1 \\
\hline High & 56 & 15.4 & 20 & 33.9 & 76 & 18.0 \\
\hline \multicolumn{7}{|l|}{ Body mass index } \\
\hline Underweight & 61 & 16.8 & 5 & 8.5 & 66 & 15.7 \\
\hline Normal & 259 & 71.3 & 37 & 62.7 & 296 & 70.1 \\
\hline Pre-obese & 33 & 9.1 & 14 & 23.7 & 47 & 11.1 \\
\hline Obese & 10 & 2.8 & 3 & 28.8 & 13 & 3.1 \\
\hline \multicolumn{7}{|l|}{ Insulin resistance } \\
\hline Yes & 67 & 18.5 & 9 & 15.3 & 76 & 18.0 \\
\hline No & 59 & 16.3 & 8 & 13.6 & 67 & 15.9 \\
\hline
\end{tabular}

Discrete data are stated as number (n) and percentage (\%) distributions

The anthropometric characteristics, physical activity scores and biochemical findings of participants are presented in Table 2.Findings are divided according to female and male participants.Especially the difference between physical activity scores of female and male participants is remarkable.

Table 2. Physical activity scores, biochemical findings and anthropometric characteristics of the participants

\begin{tabular}{|c|c|c|c|c|c|c|}
\hline \multirow[b]{2}{*}{ Anthropometry } & \multicolumn{2}{|c|}{ Female (n=363) } & \multicolumn{2}{|c|}{ Male $(n=59)$} & \multicolumn{2}{|c|}{ Total $(n=422)$} \\
\hline & Median & IQR & Median & IQR & Median & IQR \\
\hline Weight (kg) & 55.5 & 11.8 & 72.9 & 16.3 & 57.1 & 13.8 \\
\hline Height $(\mathrm{cm})$ & 163.0 & 8.0 & 176.0 & 8.0 & 164.0 & 10.0 \\
\hline BMI $\left(\mathrm{kg} / \mathrm{m}^{2}\right)$ & 21.0 & 4.1 & 23.2 & 5.2 & 21.3 & 4.2 \\
\hline Waist/hip ratio & 0.80 & 0.1 & 0.85 & 0.1 & 0.80 & 0.1 \\
\hline $\begin{array}{l}\text { Body fat percentage } \\
\text { (\%) }\end{array}$ & 26.0 & 9.0 & 14.5 & 9.1 & 24.9 & 10.3 \\
\hline \multicolumn{7}{|l|}{ Biochemical findings } \\
\hline $\begin{array}{l}\text { Total cholesterol } \\
(\mathrm{mg} / \mathrm{dL})\end{array}$ & 156.0 & 41.5 & 149.0 & 53.5 & 155.0 & 43.0 \\
\hline $\begin{array}{l}\text { HDL-cholesterol } \\
(\mathrm{mg} / \mathrm{dL})\end{array}$ & 55.0 & 19.0 & 43.0 & 14.5 & 54.0 & 19.0 \\
\hline Triglyseride (mg/dL) & 69.0 & 30.5 & 75.0 & 51.5 & 70.0 & 32.3 \\
\hline Glucose (mg/dL) & 83.0 & 15.0 & 85.0 & 16.5 & 83.0 & 15.0 \\
\hline Insulin ( $\mu$ IU/mL) & 8.2 & 5.8 & 7.3 & 5.5 & 8.0 & 5.6 \\
\hline HOMA-IR & 1.57 & 1.2 & 1.42 & 1.2 & 1.57 & 1.2 \\
\hline \multicolumn{7}{|l|}{ Physical activity } \\
\hline $\begin{array}{l}\text { MET score (MET - } \\
\text { minutes/week) }\end{array}$ & 1440.0 & 1513.0 & 2010.0 & 2561.0 & 1512.0 & 1568.4 \\
\hline
\end{tabular}

Continuous data were stated as median and interquartile range (IQR) values.Abbreviations; BMI: Body mass index, HOMA-IR: Homeostatic model assessment of insulin resistance, IQR: Interquartile range, MET: Metabolic equivalent task.

Table 3. Energy and macronutrient intakes of the participants and their meeting conditions according to the TSFNG recommendations

\begin{tabular}{|c|c|c|c|c|c|c|c|c|c|c|}
\hline & \multicolumn{3}{|c|}{ Female $(n=363)$} & \multicolumn{3}{|c|}{ Male $(n=59)$} & \multicolumn{4}{|c|}{ Total $(n=422)$} \\
\hline & Median (IQR) & $\begin{array}{l}\text { Below } \\
\mathrm{N}(\%)\end{array}$ & $\begin{array}{l}\text { Above } \\
\mathrm{N}(\%)\end{array}$ & Median (IQR) & $\begin{array}{l}\text { Below } \\
\mathrm{N}(\%)\end{array}$ & $\begin{array}{l}\text { Above } \\
\mathrm{N}(\%)\end{array}$ & $p$-value* & Median (IQR) & $\begin{array}{l}\text { Below } \\
\mathrm{N}(\%)\end{array}$ & $\begin{array}{l}\text { Above } \\
\mathrm{N}(\%)\end{array}$ \\
\hline Energy (kcal) & $1381.0(649.5)$ & $202(55.6)$ & $3(0.8)$ & $1617.4(655.0)$ & $45(76.3)$ & $0(0.0)$ & $\begin{array}{l}X^{2}=9.056 \\
p=0.011\end{array}$ & $1425.7(672.7)$ & $247(58.5)$ & $3(0.7)$ \\
\hline Protein (g) & $52.8(29.6)$ & 49 (13.5) & $118(32.5)$ & $68.6(29.5)$ & 7 (11.9) & $20(33.9)$ & $\begin{array}{l}X^{2}=0.133 \\
p=0.936\end{array}$ & $54.8(30.3)$ & $56(13.3)$ & $138(32.7)$ \\
\hline Fat (g) & $66.8(31.6)$ & NA & NA & 74.9 (37.7) & NA & NA & - & $68.0(34.0)$ & NA & NA \\
\hline Carbohydrate (g) & $139.2(95.9)$ & NA & NA & $155.2(98.4)$ & NA & NA & - & $144.1(95.2)$ & NA & NA \\
\hline Fructose (g) & $6.3(9.3)$ & NA & NA & $6.5(10.2)$ & NA & NA & - & $6.3(9.3)$ & NA & NA \\
\hline Dietary fibre (g) & $15.2(9.4)$ & 209 (57.6) & $11(3.0)$ & $16.4(12.3)$ & $34(57.6)$ & $2(3.4)$ & $\begin{array}{c}X^{2}=0.023 \\
p=0.988\end{array}$ & $15.3(9.8)$ & $243(57.6)$ & $13(3.1)$ \\
\hline Cholesterol (mg) & $200.0(209.4)$ & $183(50.4)$ & 43 (11.8) & $285.0(309.4)$ & $22(37.3)$ & $19(32.2)$ & $\begin{array}{c}X^{2}=16.839 \\
p=0.000\end{array}$ & 208.8 (1011.9) & 205 (48.6) & $62(14.7)$ \\
\hline $\begin{array}{l}\text { Saturated fatty } \\
\text { acids (g) }\end{array}$ & $21.0(13.4)$ & NA & NA & $20.6(11.7)$ & NA & NA & - & 20.9 (12.9) & NA & NA \\
\hline $\mathrm{n}-3$ (g) & $1.3(1.1)$ & $64(17.6)$ & $146(40.2)$ & $1.2(1.2)$ & $19(32.2)$ & $11(18.6)$ & $\begin{array}{c}X^{2}=12.407 \\
p=0.002\end{array}$ & $1.3(1.1)$ & 83 (19.7) & $157(37.2)$ \\
\hline$n-6$ (g) & $15.6(10.6)$ & $58(16.0)$ & 174 (47.9) & $18.1(14.9)$ & $15(25.4)$ & $22(37.3)$ & $\begin{array}{c}X^{2}=3.876 \\
p=0.144\end{array}$ & $16.0(11.0)$ & 73 (17.3) & $196(46.4)$ \\
\hline $\mathrm{n}-9$ (g) & $22.5(12.7)$ & NA & NA & $21.5(8.6)$ & NA & NA & - & $22.2(12.3)$ & NA & NA \\
\hline
\end{tabular}

* $p$-value for Pearson chi-square. Pearson's chi-square test was applied for the statistical significance of the difference between women and men who meet nutrient recommendations or not (above/below the recommendations). Abbreviations: IQR: Interquartile range, NA: none available. This abbreviation was used for data not in the TSFNG. "Below (\%) and Above (\%)" is used to indicate the percentage of respondents who are below and above recommendations. 
Table 4. Micronutrient intakes of the participants and their meeting conditions according to the TSFNG recommendations

\begin{tabular}{|c|c|c|c|c|c|c|c|c|c|c|}
\hline & \multicolumn{3}{|l|}{ Female ( $n=363$ ) } & \multicolumn{4}{|l|}{ Male $(n=59)$} & \multicolumn{3}{|l|}{ Total ( $n=422)$} \\
\hline & Median (IQR) & $\begin{array}{l}\text { Below } \\
\mathbf{N}(\%)\end{array}$ & $\begin{array}{l}\text { Above } \\
\mathrm{N}(\%)\end{array}$ & Median (IQR) & $\begin{array}{l}\text { Below } \\
N(\%)\end{array}$ & $\begin{array}{l}\text { Above } \\
N(\%)\end{array}$ & $p$-value* & Median (IQR) & $\begin{array}{l}\text { Below } \\
\mathbf{N}(\%)\end{array}$ & $\begin{array}{l}\text { Above } \\
N(\%)\end{array}$ \\
\hline $\begin{array}{l}\text { Vitamin A } \\
\text { (mcg) }\end{array}$ & $638.2(566.7)$ & $110(30.3)$ & $101(27.8)$ & $756.6(910.0)$ & $19(32.2)$ & $18(30.5)$ & $\begin{array}{l}X^{2}=0.448 \\
p=0.799\end{array}$ & 650.9 (598.8) & $129(30.6)$ & $119(28.2)$ \\
\hline $\begin{array}{l}\text { Vitamin D } \\
\text { (mcg) }\end{array}$ & $1.2(1.8)$ & $356(98.1)$ & $6(1.7)$ & $0.5(1.9)$ & $58(98.3)$ & $0(0.0)$ & $\begin{array}{l}X^{2}=3.133 \\
p=0.209\end{array}$ & $1.0(1.8)$ & 414 (98.1) & $6(1.4)$ \\
\hline $\begin{array}{l}\text { Vitamin E } \\
\text { (mg) }\end{array}$ & $16.9(10.4)$ & $75(20.7)$ & $113(31.1)$ & $19.9(13.5)$ & $9(15.3)$ & $29(49.2)$ & $\begin{array}{l}X^{2}=7.384 \\
p=0.025\end{array}$ & $17.2(11.0)$ & 84 (19.9) & $142(33.6)$ \\
\hline $\begin{array}{l}\text { Vitamin K } \\
\text { (mcg) }\end{array}$ & $264.2(197.6)$ & 7 (1.9) & $326(89.8)$ & $262.2(180.9)$ & $2(3.4)$ & $48(81.4)$ & $\begin{array}{l}X^{2}=3.600 \\
p=0.165\end{array}$ & $263.6(196.1)$ & $9(2.1)$ & $374(88.6)$ \\
\hline $\begin{array}{l}\text { Vitamin } B_{1} \\
\text { (mg) }\end{array}$ & $0.6(0.3)$ & $265(73.0)$ & $3(0.8)$ & $0.7(0.4)$ & $36(61.0)$ & $1(1.7)$ & $\begin{array}{l}X^{2}=3.688 \\
p=0.158\end{array}$ & $0.6(0.3)$ & 301 (71.3) & $4(0.9)$ \\
\hline $\begin{array}{l}\text { Vitamin } B_{2} \\
(\mathrm{mg})\end{array}$ & $1.0(0.5)$ & $59(16.3)$ & $74(20.4)$ & $1.1(0.7)$ & $13(22.0)$ & $10(16.9)$ & $\begin{array}{l}X^{2}=1.339 \\
p=0.512\end{array}$ & $1.0(0.5)$ & 72 (17.1) & $84(19.9)$ \\
\hline $\begin{array}{l}\text { Vitamin } B_{6} \\
\text { (mg) }\end{array}$ & $1.0(0.5)$ & $143(39.4)$ & $18(5.0)$ & $1.1(0.6)$ & $15(25.4)$ & $4(6.8)$ & $\begin{array}{l}X^{2}=4.275 \\
p=0.118\end{array}$ & $1.0(0.5)$ & $158(37.4)$ & $22(5.2)$ \\
\hline $\begin{array}{l}\text { Vitamin } B_{12} \\
\text { (mcg) }\end{array}$ & $3.1(2.7)$ & $90(24.8)$ & 169 (46.6) & $3.5(2.4)$ & $10(16.9)$ & $34(57.6)$ & $\begin{array}{l}X^{2}=2.798 \\
p=0.247\end{array}$ & $3.1(2.7)$ & $100(23.7)$ & $203(48.1)$ \\
\hline $\begin{array}{l}\text { Vitamin C } \\
\text { (mg) }\end{array}$ & $61.2(53.1)$ & $178(49.0)$ & 47 (12.9) & $62.6(60.0)$ & $29(49.2)$ & $8(13.6)$ & $\begin{array}{l}X^{2}=0.022 \\
p=0.989\end{array}$ & $61.2(55.2)$ & 207 (49.1) & $55(13.0)$ \\
\hline $\begin{array}{l}\text { Folate } \\
\text { (mcg) }\end{array}$ & $190.0(100.5)$ & $292(80.4)$ & $1(0.3)$ & $223.2(133.1)$ & $38(64.4)$ & $2(3.4)$ & $\begin{array}{l}X^{2}=12.609 \\
p=0.002\end{array}$ & $193.1(104.1)$ & $330(78.2)$ & $3(0.7)$ \\
\hline $\begin{array}{l}\text { Sodium } \\
\text { (mg) }\end{array}$ & $3241.0(1803.5)$ & $33(9.1)$ & $184(50.7)$ & 3947.8 (2595.7) & $6(10.2)$ & $37(62.7)$ & $\begin{array}{l}X^{2}=3.734 \\
p=0.155\end{array}$ & $3296.2(1946.5)$ & $39(9.2)$ & $221(52.4)$ \\
\hline $\begin{array}{l}\text { Potassium } \\
\text { (mg) }\end{array}$ & 1707.0 (863.8) & NA & NA & $1928.1(854.6)$ & NA & NA & - & $1735.1(864.3)$ & NA & NA \\
\hline $\begin{array}{l}\text { Calsium } \\
\text { (mg) }\end{array}$ & $560.1(335.3)$ & $239(65.8)$ & $3(0.8)$ & $532.5(406.4)$ & $39(66.1)$ & $0(0.0)$ & $\begin{array}{l}X^{2}=0.493 \\
p=0.782\end{array}$ & 559.5 (343.8) & 278 (65.9) & $3(0.7)$ \\
\hline $\begin{array}{l}\text { Magnesium } \\
\text { (mg) }\end{array}$ & $217.8(108.6)$ & $162(44.6)$ & $10(2.8)$ & $242.6(114.3)$ & $32(54.2)$ & $3(5.1)$ & $\begin{array}{l}X^{2}=3.334 \\
p=0.189\end{array}$ & $221.3(107.5)$ & 194 (46.0) & $13(3.1)$ \\
\hline $\begin{array}{l}\text { Phosphor } \\
\text { (mg) }\end{array}$ & $903.1(380.6)$ & $18(5.0)$ & $164(45.2)$ & $1088.0(479.0)$ & $3(5.1)$ & $37(62.7)$ & $\begin{array}{l}X^{2}=6.616 \\
p=0.037\end{array}$ & $918.0(410.2)$ & $21(5.0)$ & 201 (47.6) \\
\hline Iron (mg) & $8.6(4.2)$ & $305(84.0)$ & $0(0.0)$ & $10.0(4.9)$ & $18(30.5)$ & $11(18.6)$ & $\begin{array}{l}X^{2}=116.3 \\
p=0.000\end{array}$ & $8.7(4.3)$ & $323(76.5)$ & $11(2.6)$ \\
\hline Zinc (mg) & $7.5(3.9)$ & $144(39.7)$ & $12(3.3)$ & $9.7(4.2)$ & $13(22.0)$ & $5(8.5)$ & $\begin{array}{l}X^{2}=8.951 \\
p=0.011\end{array}$ & $7.7(4.1)$ & $157(37.2)$ & $17(4.0)$ \\
\hline
\end{tabular}

* $p$-value for Pearson chi-square. Pearson's chi-square test was applied for the statistical significance of the difference between women and men who meet nutrient recommendations or not (above/below the recommendations). Abbreviations: IQR: Interquartile range, NA: none available. This abbreviation was used for data not in the TSFNG. "Below (\%) and Above (\%)" is used to indicate the percentage of respondents who are below and above recommendations.

The median values of protein, fat and carbohydrate percentages of all participants are as follows; $16.0 \%$, $42.0 \%$ and $43.0 \%$. While these values for women were $16.0 \%, 42.0 \%$ and $43.0 \%$, respectively; It was found to be $17.0 \%, 40.0 \%$ and $41.0 \%$ in males.Energy, macro-and micronutrient intakes of the participants are given in Tables 3 and 4 . Accordingly, the energy intake of $54.5 \%$ of the participants was below TSFNG. Besides, $32.7 \%$ of the participants had high intakes of protein, and $13.3 \%$ low the recommended. The protein intakes of male participants were higher than female $(p<0.05)$. The median percentage contribution of calories from protein, carbohydrate, and fat, were $16.0 \%, 43.0 \%$ and $42.0 \%$, respectively. Cholesterol intakes were higher in men than in women $(p<0.05)$. According to micronutrients, women's intakes of vitamins E and B6, folic acid, sodium, phosphorus, iron and zinc were less than men $(p<0.05)$. Folic acid intake of $80.4 \%$ of women was found below the recommended one. It was determined that $52.4 \%$ of all participants had high sodium intake.

In Table 5, HDL-C was shown negatively correlated with $\mathrm{BMI}$ and WHR $(p<0.05)$. On the other hand, fasting plasma insulin level and HOMA-IR have a positive correlation with BMI, WHR and body fat\% $(p<0.05)$. Triglyceride levels were positively correlated with weight, BMI and WHR $(p<0.05)$. There was no significant relationship between physical activity levels and biochemical findings $(p>0.05)$. In Table 5 , the HDL-C level has a negative correlation with total fatty acid and saturated fatty acid (SFA) intake $(p<0.05)$. Omega-3 intakes of the participants were negatively correlated with fasting plasma insulin and HOMA-IR $(p<0.05)$. 
Table 5. Relationship between biochemical findings and anthropometric measurements, physical activity scores, energy and nutrient intake of the participants

\begin{tabular}{|c|c|c|c|c|c|c|c|c|c|c|c|c|}
\hline \multirow[b]{2}{*}{ Anthropometry } & \multicolumn{2}{|c|}{ Total cholesterol } & \multicolumn{2}{|c|}{ HDL cholesterol } & \multicolumn{2}{|c|}{ Glucose } & \multicolumn{2}{|c|}{ Insulin } & \multicolumn{2}{|c|}{ Triglyseride } & \multicolumn{2}{|c|}{ HOMA-IR } \\
\hline & r & $p$ & r & $p$ & r & $p$ & r & $p$ & r & $p$ & r & $p$ \\
\hline Weight (kg) & .024 & 0.624 & -.295 & 0.001 & -.070 & 0.152 & .134 & 0.006 & .137 & 0.005 & .156 & 0.001 \\
\hline BMI $\left(\mathrm{kg} / \mathrm{m}^{2}\right)$ & .055 & 0.256 & -.227 & 0.001 & -.072 & 0.138 & .123 & 0.012 & .148 & 0.002 & .171 & 0.01 \\
\hline Waist/hip ratio & .076 & 0.122 & -.165 & 0.001 & -.067 & 0.169 & .231 & 0.001 & .177 & 0.001 & .229 & 0.01 \\
\hline Body fat percentage (\%) & .086 & 0.079 & -.005 & 0.915 & -.096 & 0.050 & .199 & 0.001 & .013 & 0.797 & .162 & 0.001 \\
\hline \multicolumn{13}{|l|}{ Physical activity } \\
\hline $\begin{array}{l}\text { MET score (MET - } \\
\text { minutes/week) }\end{array}$ & .016 & 0.739 & -.083 & 0.090 & .036 & 0.465 & -.072 & 0.142 & .027 & 0.582 & .011 & 0.819 \\
\hline \multicolumn{13}{|c|}{ Energy, macro ve micro nutrients } \\
\hline Energy (kcal) & -.012 & 0.805 & -.050 & 0.301 & .030 & 0.545 & -.054 & 0.265 & .036 & 0.465 & .031 & 0.532 \\
\hline Protein (g) & -.065 & 0.183 & -.099 & 0.042 & -.056 & 0.250 & -.078 & 0.111 & -.040 & 0.411 & -.044 & 0.367 \\
\hline Protein (\%) & -.077 & 0.112 & -.062 & 0.205 & -.120 & 0.014 & -.039 & 0.421 & -.129 & 0.008 & -.125 & 0.010 \\
\hline Fat (g) & -.069 & 0.156 & -.097 & 0.046 & -.026 & 0.591 & -.014 & 0.770 & -.009 & 0.847 & -.003 & 0.944 \\
\hline Fat (\%) & -.052 & 0.290 & .019 & 0.695 & -.044 & 0.366 & .057 & 0.239 & -.117 & 0.017 & -.093 & 0.056 \\
\hline Carbohydrate (g) & -.003 & 0.955 & -.059 & 0.225 & .048 & 0.325 & -.033 & 0.501 & .095 & 0.052 & .088 & 0.070 \\
\hline Carbohydrate (\%) & .067 & 0.168 & .016 & 0.749 & .077 & 0.112 & -.028 & 0.563 & .141 & 0.004 & .128 & 0.009 \\
\hline Fructose (g) & -.004 & 0.928 & -.065 & 0.184 & .086 & 0.079 & .080 & 0.100 & .007 & 0.891 & .031 & 0.529 \\
\hline Dietary fibre (g) & -.083 & 0.090 & -.124 & 0.011 & .029 & 0.553 & -.039 & 0.429 & -.006 & 0.904 & -.025 & 0.613 \\
\hline Cholesterol (mg) & .017 & 0.727 & -.025 & 0.613 & -.070 & 0.151 & -.023 & 0.640 & -.186 & 0.000 & -.168 & 0.001 \\
\hline Saturated fatty acids (g) & -.080 & 0.100 & -.116 & 0.017 & -.059 & 0.227 & .001 & 0.985 & -.081 & 0.095 & -.074 & 0.131 \\
\hline$n-3$ (g) & -.017 & 0.733 & -.028 & 0.561 & -.087 & 0.075 & -.022 & 0.657 & -.147 & 0.002 & -.136 & 0.005 \\
\hline $\mathrm{n}-6$ (g) & .068 & 0.160 & .027 & 0.579 & .033 & 0.500 & .061 & 0.213 & .050 & 0.310 & .061 & 0.211 \\
\hline n-9 (g) & -.030 & 0.539 & -.024 & 0.627 & -.026 & 0.600 & -.013 & 0.796 & -.074 & 0.131 & -.061 & 0.210 \\
\hline Vitamin A (mcg) & -.049 & 0.315 & -.107 & 0.028 & -.027 & 0.581 & -.026 & 0.599 & -.158 & 0.001 & -.166 & 0.001 \\
\hline Vitamin D (mcg) & .006 & 0.903 & .029 & 0.547 & -.019 & 0.692 & -.036 & 0.467 & -.088 & 0.072 & -.085 & 0.081 \\
\hline Vitamin E (mg) & -.052 & 0.285 & -.124 & 0.011 & .029 & 0.546 & -.018 & 0.713 & .080 & 0.231 & .051 & 0.298 \\
\hline Vitamin K (mcg) & -.011 & 0.815 & -.018 & 0.712 & -.022 & 0.657 & .035 & 0.474 & -.054 & 0.269 & -.051 & 0.300 \\
\hline Vitamin $B_{1}(\mathrm{mg})$ & -.139 & 0.004 & -.153 & 0.002 & -.050 & 0.302 & -.051 & 0.293 & -.030 & 0.537 & -.051 & 0.298 \\
\hline Vitamin $B_{2}(m g)$ & -.044 & 0.367 & -.030 & 0.541 & -.020 & 0.683 & .003 & 0.953 & -.104 & 0.033 & -.096 & 0.050 \\
\hline Vitamin $B_{6}(\mathrm{mg})$ & -.133 & 0.006 & -.108 & 0.026 & -.044 & 0.363 & -.031 & 0.520 & -.013 & 0.794 & -.029 & 0.546 \\
\hline Vitamin $\mathbf{B}_{12}(\mathrm{mcg})$ & -.051 & 0.295 & -.145 & 0.003 & .022 & 0.651 &.$- * 43$ & 0.378 & -.036 & 0.461 & -.043 & 0.378 \\
\hline Vitamin C (mg) & -.001 & 0.983 & -.009 & 0.856 & .024 & 0.630 & .045 & 0.359 & -.092 & 0.059 & -.084 & 0.084 \\
\hline Folate (mcg) & -.098 & 0.044 & -.112 & 0.022 & -.044 & 0.368 & -.037 & 0.453 & -.121 & 0.013 & -.131 & 0.007 \\
\hline Sodium (mg) & -.025 & 0.607 & -.054 & 0.273 & .042 & 0.393 & -.010 & 0.840 & .026 & 0.598 & .030 & 0.545 \\
\hline Potassium (mg) & -.109 & 0.025 & -.114 & 0.019 & -.015 & 0.765 & -.038 & 0.438 & -.027 & 0.574 & -.047 & 0.337 \\
\hline Calsium (mg) & -.011 & 0.820 & .021 & 0.664 & .027 & 0.579 & .021 & 0.671 & -.039 & 0.422 & -.032 & 0.512 \\
\hline Magnesium (mg) & -.117 & 0.016 & -.145 & 0.003 & .016 & 0.748 & -.013 & 0.791 & .011 & 0.829 & -.004 & 0.936 \\
\hline Phosphor (mg) & -.076 & 0.121 & -.102 & 0.036 & -.025 & 0.613 & -.031 & 0.531 & -.047 & 0.335 & -.054 & 0.268 \\
\hline Iron (mg) & -.095 & 0.051 & -.165 & 0.001 & -.001 & 0.982 & -.064 & 0.187 & -.038 & 0.438 & -.054 & 0.267 \\
\hline Zinc (mg) & -.054 & 0.266 & -.137 & 0.005 & .011 & 0.828 & -.044 & 0.366 & -.041 & 0.402 & -.043 & 0.383 \\
\hline
\end{tabular}

Spearmen correlation test. Abbreviations; BMI: Body mass index, HOMA-IR: Homeostatic model assessment of insulin resistance, MET: Metabolic equivalent task. 
Table 6. Relationship between anthropometric measurements of the participants and their energy, macro and micronutrient intakes

\begin{tabular}{|c|c|c|c|c|c|c|}
\hline & \multicolumn{2}{|c|}{ Body weight } & \multicolumn{2}{|c|}{$\begin{array}{l}\text { Body mass } \\
\text { index }\end{array}$} & \multicolumn{2}{|c|}{$\begin{array}{l}\text { Waist-to-hip } \\
\text { ratio }\end{array}$} \\
\hline & $r$ & $p$ & $r$ & $p$ & $r$ & $p$ \\
\hline Energy (kcal) & .003 & .945 & -.064 & .188 & -.056 & .255 \\
\hline Protein (g) & .076 & .121 & .024 & .624 & .047 & .336 \\
\hline Protein (\%) & .112 & .021 & .118 & .015 & .104 & .032 \\
\hline Fat (g) & .022 & .654 & -.023 & .637 & -.018 & .708 \\
\hline Fat (\%) & .068 & .161 & .130 & .008 & .021 & .672 \\
\hline Carbohydrate (g) & -.074 & .130 & -.156 & .001 & -.067 & .169 \\
\hline $\begin{array}{l}\text { Carbohydrate } \\
\text { (\%) }\end{array}$ & -.096 & .049 & -.155 & .001 & -.068 & .166 \\
\hline Fructose (g) & .000 & .992 & .005 & .917 & -.010 & .831 \\
\hline Dietary fibre (g) & -.042 & .387 & -.094 & .054 & -.153 & .002 \\
\hline Cholesterol (mg) & .105 & .032 & .085 & .080 & .037 & .450 \\
\hline $\begin{array}{l}\text { Saturated fatty } \\
\text { acids (g) }\end{array}$ & .036 & .463 & -.019 & .698 & -.046 & .351 \\
\hline $\mathrm{n}-3$ (g) & .000 & .989 & -.021 & .671 & -.055 & .262 \\
\hline$n-6$ (g) & -.002 & .973 & -.040 & .408 & .080 & .102 \\
\hline$n-9(g)$ & .005 & .920 & -.060 & .217 & -.026 & .592 \\
\hline Vitamin A (mcg) & .080 & .099 & .070 & .150 & -.071 & .144 \\
\hline Vitamin D (mcg) & -.053 & .278 & -.044 & .366 & -.146 & .003 \\
\hline Vitamin E (mg) & .009 & .849 & -.039 & .427 & .033 & .500 \\
\hline Vitamin K (mcg) & -.059 & .225 & -.059 & .230 & -.096 & .048 \\
\hline Vitamin $B_{1}(\mathrm{mg})$ & .027 & .580 & -.039 & .426 & -.052 & .289 \\
\hline Vitamin $B_{2}(\mathrm{mg})$ & .044 & .364 & .003 & .953 & -.041 & .398 \\
\hline Vitamin $\mathrm{B}_{6}(\mathrm{mg})$ & -.007 & .880 & -.057 & .246 & -.048 & .322 \\
\hline Vitamin $B_{12}(\mathrm{mcg})$ & .047 & .335 & .061 & .213 & .020 & .675 \\
\hline Vitamin C (mg) & .003 & .951 & .021 & .666 & -.046 & .351 \\
\hline Folate (mcg) & .021 & .660 & -.005 & .914 & -.104 & .033 \\
\hline Sodium (mg) & .054 & .267 & .040 & .410 & .025 & .612 \\
\hline Potassium (mg) & -.015 & .754 & -.042 & .384 & -.056 & .255 \\
\hline Calsium (mg) & .019 & .695 & .011 & .829 & -.027 & .587 \\
\hline Magnesium (mg) & .008 & .864 & -.040 & .414 & -.101 & .038 \\
\hline Phosphor (mg) & .042 & .393 & -.014 & .781 & -.030 & .539 \\
\hline Iron (mg) & .052 & .282 & -.017 & .728 & -.053 & .275 \\
\hline Zinc (mg) & .076 & .120 & .005 & .911 & .026 & .588 \\
\hline
\end{tabular}

Spearmen correlation test.

\section{DISCUSSION}

Various cardiometabolic risk factors such as obesity, increased $\mathrm{BMI}$ and high fasting plasma glucose levels constitute the early onset of chronic conditions in adulthood. So proper diet and adequate physical activity are of great importance in young adulthood (19). In this study, the biochemical findings, food consumptions, physical activity levels and anthropometric properties of university students were evaluated.

The fact that the majority of the participants (70.1\%) were within the normal $\mathrm{BMI}$ range, according to WHO classifications, is similar to a study conducted with university students (10). However, in some studies, it was shown that the frequency of normal BMI is higher than in this study $(20,21,22)$. In this study, the frequency of underweight women was higher than men, while the frequency of preobese and obese individuals was found to be lower in female participants. Similarly, in a study in Spain, it was reported that the higher frequency of underweight, because of the recent increase in eating disorders and restrictive food intake behaviour in women (10).

In this study, body fat $\%$ was found to be $26.1 \%$ in women and $16.1 \%$ men. In two studies with students studying on health, in one of them, the body fat\% in women $(24.1 \%)$ was found to be similar to this study (10), while it was observed to be lower (21.9\%) in the other one. Unlike this study, a study with university students in Turkey reported that the average body fat\% in women was $30 \%$ (23). The WHR of both men and women were higher than in various studies $(23,24,25,26)$. In a study conducted with 968 participants in Brazil, a similar WHR was reported in men as in this study (27).

The frequency of women with a high level of physical activity was found to be lower than menin this study. Similarly, a study with 704 participants reported that $21.6 \%$ of women had a high activity level, while this frequency was $40.0 \%$ for men (28). It was determined that physical activity levels of studies with young adults using different physical activity measurement tools differ with this study $(21,22,29)$.

Anthropometric measurement results were found to be positively correlated with triglyceride and fasting plasma insulin levels while negatively with HDL-C levels. Similarly, Arizaga et al. (2018) found that BMI and body fat\% were positively correlated with triglyceride levels (25). Likewise, in a study in Turkey showed that there is a significant relationship between $\mathrm{BMI}$ and triglyceride levels (23).In a study conducted to determine cardiovascular risk factors in students, it was reported that BMI and WHR were positively correlated with $\mathrm{HDL}-\mathrm{C}$, triglyceride and fasting plasma insulin levels (27). Similarly, a study showed that BMI was negatively correlated with HDL-C level and positively correlated with triglyceride, total cholesterol levels (30).

Although the frequency of $I R$, determined according to HOMA-IR, appears to be higher in women than men, it was observed that the difference was not significant. Similarly, a study reported that although the HOMA-IR value was higher in women, the difference was not significant (27). This study showed that there is a significant relationship between HOMA-IR level and anthropometric measurements. Similarly, de Carvalho et al. (2015) showed a positive relationship between HOMA-IR value and BMI, WHR in women, while a significant relationship was found withonly WHR in men (27). 
It was determined that more than half of the participants' energy intake was below the recommendation when the food consumption was evaluated. Therefore, it was observed similarity with Turkey Nutrition and Health Survey 2019 (TNHS 2019) in energy intakes. TNHS 2019 includes the data about the food consumption of the Turkish adults (18 years and over)(31). Similarly, in some studies with university students, it was reported that the energy intake was below the recommended $(10,23,32)$.

This study showed that participants' carbohydrate and dietary fibre intakes were lower than recommended while their fat intakes were high. It has been found that dietary fibre intakes are also below TNHS 2019 (31).The reason is that university students have widespread Western-style dietary habits. A high-fat, low-fibre diet, especially high intake of SFA, contributes to inflammation and lays the ground for various chronic diseases (33). Also, this study showed a positive correlation between total fat, SFA intake and total cholesterol level. Contrary to this, higher carbohydrate and lower fat intakes were reported in various studies $(23,32$, 34). Similarly, in a study evaluating compliance with the Mediterranean diet, university students' dietary fibre intake was found to be below the recommended while SFA intake was high (35).

In this study, the polyunsaturated fatty acids (PUFA) of participants were found above the recommended, especially in females, similar to the TNHS 2019 data (31). Also, omega-3 intake is negatively related to fasting plasma insulin level and IR. Cholesterol intakes above the recommended were observed significantly higher in men than in women. Epidemiological studies show that populations with high intakes of omega-3 fatty acids have a low cardiovascular risk. PUFA consumption is known to have anti-arrhythmic and cardioprotective effects (36). Correa-Rodriguez et al. (2018) showed that university students' PUFA intake was similarly higher than the recommended. Besides, similar to this study, when the fatty acid profile of the diet was examined, SFA intake was reported higher than monounsaturated fatty acid intake (10).

The results also showed that the micronutrient content of the diet was generally insufficient. It was observed that the vitamins B1, B6 and folate intakes were also below the TNHS 2019 data (31). In a study in Turkey, the vitamins B1, B6 and folate intakes of the students were below the recommendations, other vitamins were taken adequately (37). In another study, it was also reported that folate intake was insufficient (38). Similarly, Hervás et al. (2018), evaluating the relationship between nutrition and bone health,showed that the calcium intake in university students did not meet the recommendations, although it was higher than this study (39). Unlike our study, in a study with the general Spanish population, $71.8 \%$ of the adults were had sufficient vitamin B1 intake (40). Vitamin C, one of the important antioxidants, is known to play a role in cholesterol metabolism as well as a cofactor in many biochemical reactions such as the synthesis of collagen, carnitine and catecholamines (41). B vitamins (especially B6, B12 and folate) are among the important regulators of homocysteine metabolism. Consuming foods rich in B vitamins or taking supplements reduces the risk of developing vascular diseases such as atherosclerosis and stroke by lowering the concentrations of circulating homocysteine (42). Nutrition is critical in the development and maintenance of bone mass, and adequate intakes of magnesium, calcium, vitamins C, D and $\mathrm{K}$ are essential for maintaining bone health.Inadequate and imbalanced diet seen in young adults leads to deficiencies in vitamin and mineral intake (39). Vitamin B1, which plays a role in energy metabolism and the growth and development of cells, is found richin foods such as bread, cereals, legumes, meat, liver, and fish (40). Therefore, based on our results, it is thought that insufficient intake of carbohydrate resulted in insufficient vitamin B1 intake.

Without gender difference, in this study, dietary intake of calcium, magnesium and zinc was shown to be insufficient. But intake of iron was observed to be below the recommended in only women. Similarly, Şanlıer (2005) reported that these minerals were below the recommended for both men and women, but phosphorus was taken above the recommended one. Also, iron intake was shown to be significantly higher in men than in women (23). Calcium plays anessentialrole in signal transduction in cells as well as bone health. Magnesium, which acts as a cofactor in many enzymatic reactions, has critical functions in muscle contraction, glucose utilization, synthesis of nucleic acids, fats and proteins (43). Similar to TNHS 2019 and Correa-Rodriguez et al. (2018), in this study, participants' dietary sodium and phosphorus intake was found to be quite high $(10,31)$. It is known that high sodium intake,threatening cardiovascular health, is an independent risk factor for hypertension (44). Phosphorus, an important component of nucleic acids, plays an essential role in the structure of high-energy nucleotides and cellular signal transmission (43).

The limitations of this study can be explained that not addressing the effect of the living conditions of the participants on the outcome, taking a 24-hour dietary recall, not taking into account the plasma levels of vitamins and minerals while evaluating the dietary intake of micronutrients.

However, supporting this study with the biochemical findings of participants, making the anthropometric measurements by the researcher and the high number of participants can be considered as the strengths of this study.

\section{CONCLUSION}

This study emphasizes that factors such as inadequate and imbalanced diet, high BMI, WHR, body fat percentage have negative effects on the lipid profile; and these factors are associated with IR in young adults. It also shows that participants'total fat, SFA, sodium, phosphorus intakes were high; and carbohydrate, dietary fibre, vitamin $D$, folic acid, calcium, iron and zinc intakes were insufficient. For this reason, it is thought that developing nutrition strategies, 
providing nutrition counselling, preventing nutrients deficiencies and creating balanced nutrition habits are needed to improve the nutrition habits of university students. The provision of education and counselling services, the development of campus facilities will ensure all students are physically active. Thanks to these facilities, positive changes are expected in the results of university students' anthropometric measurements. Thus, they will be protected from cardiometabolic risks during the university period when lifestyle habits are acquired.

\section{Acknowledgements}

This work has been supported by Marmara University Scientific Research Projects Coordination Unit under grant number SAG-C-YLP-070.617.0344.

\section{REFERENCES}

[1] Yahia N, Wang D, Rapley M, Dey R. Assessment of weight status, dietary habits and beliefs, physical activity, and nutritional knowledge among university students. Perspect Public Health 2016; 136(4):231-244.

[2] Nelson MC, Story M, Larson NI, Neumark-Sztainer D, Lytle LA. Emerging adulthood and college-aged youth: an overlooked age for weight-related behavior change. Obesity (Silver Spring) 2008; 16(10):2205-2211.

[3] Levy SB, Klimova TM, Zakharova RN, Federov Al, Fedorova VI, Baltakhinova ME, Leonard WR. Brown adipose tissue, energy expenditure, and biomarkers of cardio-metabolic health among the Yakut (Sakha) of northeastern Siberia. Am J Hum Biol 2018; 30(6):e23175.

[4] Daneshzad E, Shab-Bidar S, Mohammadpour Z, Djafarian K. Effect of anthocyanin supplementation on cardio-metabolic biomarkers: A systematic review and meta-analysis of randomized controlled trials. Clin Nutr 2019; 38(3):1153-1165.

[5] Gayoso-Diz P, Otero-González A, Rodriguez-Alvarez MX, Gude F, García F, De Francisco A, Quintela AG. Insulin resistance (HOMA-IR) cut-off values and the metabolic syndrome in a general adult population: effect of gender and age: EPIRCE cross-sectional study. BMC Endocr Disord 2013; 13:47.

[6] Zaccagni L, Barbieri D, Gualdi-Russo E. Body composition and physical activity in Italian university students. J Transl Med 2014; 12:120.

[7] Kowalcze K, Turyk Z, Drywień M. Nutrition of students from dietetics profile education in the Siedlce University of Natural Sciences and Humanities compared with students from other academic centres. Rocz Panstw Zakl Hig 2016; 67(1):51-58.

[8] Plotnikoff RC, Costigan SA, Williams RL, Hutchesson MJ, Kennedy SG, Robards SL, Allen J, Collins CE, Callister R, Germov J.Effectiveness of interventions targeting physical activity, nutrition and healthy weight for university and college students: a systematic review and meta-analysis. Int J Behav Nutr Phys Act 2015;12:45.

[9] Belogianni K, Ooms A, Ahmed H, Nikoletou D, Grant R, Makris $D$, Moir HJ. Rationale and design of an online educational program using game-based learning to Improve nutrition and physical activity outcomes among university students in the United Kingdom. J Am Coll Nutr 2019; 38(1):23-30.
[10] Correa-Rodríguez M, Pocovi G, Schmidt-RioValle J, GonzálezJiménez E, Rueda-Medina B. Assessment of dietary intake in Spanish university students of health sciences. Endocrinol Diabetes Nutr 2018; 65(5): 265-273.

[11] Hamulka J, Wadolowska L, Hoffmann M, Kowalkowska J, Gutkowska K. Effect of an education program on nutrition knowledge, attitudes toward nutrition, diet quality, lifestyle, and body composition in Polish Teenagers. The ABC of healthy eating project: Design, protocol, and methodology. Nutrients. 2018;10(10):1439.

[12] Liu AG, Ford NA, Hu FB, Zelman KM, Mozaffarian D, KrisEtherton PM. A healthy approach to dietary fats: understanding the science and taking action to reduce consumer confusion. Nutrition Journal. 2017; 16(53): 1-15.

[13] Eliat-Adar S, Sinai T, Yosefy C, Henkin Y. Nutritional recommendations for cardiovascular disease prevention. Nutrients. 2013; 5(9): 3646-3683.

[14] Nowak PF, Bożek A, Blukacz M. Physical activity, sedentary behavior, and quality of life among university students. Biomed Res Int. 2019; 2019:9791281.

[15] Castro O, Bennie J, Vergeer I, Bosselut G, Biddle SJH. How sedentary are university students? A systematic review and meta-analysis. Prev Sci 2020; 21(3):332-343.

[16] Ashok P, Kharche JS, Raju R, Godbole G. Metabolic equivalent task assessment for physical activity in medical students. Natl J Physiol Pharm Pharmacol. 2017;7(3):236.

[17] Craig $C L$, Marshall AL, Sjöström $M$, Bauman $A E$, Booth $M L$, Ainsworth BE, Pratt M, Ekelund U, Yngve A, Sallis JF, Oja P. International physical activity questionnaire: 12-Country reliability and validity. Med Sci Sports Exerc 2003; 35(8): 13811395.

[18] Besler HT, Rakıcıoğlu N, Ayaz A, Büyüktuncer-Demirel Z, Gökmen-Özel H, Samur FG, et al. Türkiye'ye Özgü Besin ve Beslenme Rehberi [Turkey Specific Food and Nutrition Guide]. Ankara: Merdiven Reklam Tanıtım; 2015. (Turkish)

[19] Akseer N, Al-Gashm S, Mehta S, Mokdad A, Bhutta ZA. Global and regional trends in the nutritional status of young people: a critical and neglected age group. Ann N Y Acad Sci 2017; 1393(1):3-20.

[20] Yılmaz HÖ, Ayhan NY. Hemşirelik öğrencilerinin bazı beslenme alışkanlıklarının değerlendirilmesi [Evaluation of some nutritional habits of nursing students]. Gümüşhane Üniversitesi Sağlık Bilimleri Dergisi 2017; 6(1): 17-24. (Turkish)

[21] Tözün M, Sözmen MK, Babaoğlu $A B$. Türkiye'nin batısında bir üniversitenin sağlık ile ilişkili okullarında beslenme alışkanlıkları ve bunun obezite, fizik aktivite ve yaşam kalitesi ile ilişkisi [Nutritional habits in health-related departments of a university in the west of Turkey and its relation to obesity, physical activity and quality of life]. Türk Dünyası Uygulama ve Araştırma Merkezi Halk Sağ|ığı Dergisi. 2017; 2(1):1-16. (Turkish)

[22] Soyuer F, Ünalan D, Elmalı F. Normal ağılıklı ve obez üniversite öğrencilerinde fiziksel aktivite [Physical activity in normalweight and obese university students]. Uluslararası Insan Bilimleri Dergisi. 2010; 9(2): 862-872. (Turkish)

[23] Şanlıer N. Gençlerde biyokimyasal bulgular, antropometrik ölçümler, vücut bileşimi, beslenme ve fiziksel aktivite durumlarının değerlendirilmesi [Biochemical findings, anthropometric measurements, body composition, nutrition and physical activity status in young people]. Gazi Eğitim Fakültesi Dergisi. 2005; 25(3): 47-73. (Turkish) 
[24] Açık M, Çakıroğlu FP. Yurtta kalan üniversiteli kız öğrencilerde metabolik sendrom risk faktörlerinin değerlendirilmesi [Evaluation of metabolic syndrome risk factors in female students staying in dorms]. Dicle Tıp Dergisi 2017; 44(4): 305314. (Turkish)

[25] Arizaga IZ, Atucha AF, Kortajarena M, Torres-Unda J, Irazusta A, Fraile-Bermudez AB. Associations of anthropometric characteristics, dietary habits, and aerobic capacity with cardiovascular risk factors of health-science students. Front Nutr 2018; 5: 1-10.

[26] Beaudry KM, Ludwa IA, Thomas AM, Ward WE, Falk B, Josse AR. First-year university is associated with greater body weight, body composition and adverse dietary changes in males than females. PLoS One 2019; 14(7):e0218554.

[27] de Carvalho CA, Fonseca PC, Barbosa JB, Machado SP, dos Santos AM, da Silva AA. The association between cardiovascular risk factors and anthropometric obesity indicators in university students in São Luís in the State of Maranhão, Brazil. Cien Saude Colet. 2015; 20(2):479-490.

[28] Genç A, Şener Ü, Karabacak H, Üçok K. Kadın ve erkek genç erişkinler arasında fiziksel aktivite ve yaşam kalitesi farklılıklarının araştırılması [Investigation of differences in physical activity and quality of life between male and female young adults]. Kocatepe Tıp Dergisi 2011; 12(3): 145-150. (Turkish)

[29] Yıldız A, Tarakcı D, Mutluay FK. Genç erişkinlerde fiziksel aktivite düzeyi ile vücut kompozisyonu ilişkisi: pilot çalışma [The relationship between physical activity level and body composition in young adults: a pilot study]. HSP 2015; 2(3): 297-305. (Turkish)

[30] Ruano C, Lucumi E, Albán J, Arteaga S, Fors M. Obesity and cardio-metabolic risk factors in Ecuadorian university students. First report, 2014-2015. Diabetes Metab Syndr 2018; 12(6):917-921.

[31] Türkiye Beslenme ve Sağlık Araştırması 2019 [Turkey Nutrition and Health Survey 2019]. Sağlık Araştırmaları Genel Müdürlüğü, Sağlık Bakanlığı, https://hsgm.saglik.gov.tr/depo/birimler/ saglikli-beslenme-hareketli-hayat-db/Yayinlar/kitaplar/TBSA_ RAPOR_KITAP_20.08.pdf, Erişim tarihi: 01.11.2020.

[32] Barker ME, Blain RJ, Russell JM. The influence of academic examinations on energy and nutrient intake in male university students. Nutrition Journal 2015; 14(98): 1-7.

[33] Neuhouser ML. The importance of healthy dietary patterns in chronic disease prevention. Nutr Res 2019; 70:3-6.
[34] 34. Kızıltan G, Karabudak E, Ünver S, Sezgin E, Ünal A. Nutritional status of university students with binge eating disorder. Ankara Üniversitesi Tıp Fakültesi Mecmuası. 2005; 58(4):167-171.

[35] García-Meseguer MJ, Burriel FC, García CV, Serrano-Urrea R. Adherence to Mediterranean diet in a Spanish university population. Appetite 2014; 78:156-164.

[36] Medenwald D, Kluttig A, Lacruz ME, Schumann J. Serum dietary fatty acids and coronary heart disease risk - A nested case-control-study within the CARLA cohort. Nutr Metab Cardiovasc Dis 2019; 29(2):152-158.

[37] Rakicioglu N, Yildiz EA. Energy and nutrient intake and food patterns among Turkish university students. Nutrition Research and Practice 2011; 5(2):117-123.

[38] Gallo L, Moritz K, Akison L. Nutrient intake, physical activity levels, and metabolic status in Australian University biomedical students. CDN 2020; 4(2): 1404.

[39] Hervás G, Ruiz-Litago F, Irazusta J, Fernández-Atutxa A, FraileBermúdez AB, Zarrazquin I. Physical activity, physical fitness, body composition, and nutrition are associated with bone status in university students. Nutrients 2018 10(1):61.

[40] Mielgo-Ayuso J, Aparicio-Ugarriza R, Olza J, Aranceta-Bartrina J, Gil Á, Ortega RM, Serra-Majem L, Varela-Moreiras G, González-Gross M. Dietary intake and food sources of niacin, riboflavin, thiamin and vitamin $B_{6}$ in a representative sample of the Spanish population. The ANIBES Study. Nutrients 2018;10(7): 846.

[41] Olza J, Aranceta-Bartrina J, González-Gross M, Ortega RM, Serra-Majem, L, Varela-Moreiras G, Gil Á. Reported dietary intake and food sources of zinc, selenium, and vitamins $A, E$ and $C$ in the Spanish population: Findings from the ANIBES study. Nutrients 2017; 6;9(7):697.

[42] Chen L, Li Q, Fang X, Wang X, Min J, Wang F. Dietary intake of homocysteine metabolism-related B-vitamins and the risk of stroke: a dose-response meta-analysis of prospective studies. Adv Nutr 2020; 00:1-19.

[43] Malara M, Tkaczyk J, Kęska A, Lutosławska G, Mazurek K. Calcium, magnesium and phosphorus dietary intake in active and sedentary Polish students. Biomedical Human Kinetics 2017;9(1), 140-145.

[44] O'Donnell M, Mente A, Yusuf S. Sodium intake and cardiovascular health. Circ Res 2015; 116(6):1046-1057.

How to cite this article: Icen H, Gunes F. The Relationship Between University Students' Nutritional Status, Cardio-Metabolic Biomarkers and Physical Activity Levels. Clin Exp Health Sci 2021; 11: 495-503. DOI: 10.33808/clinexphealthsci.820145 